\title{
AURC Infinity Predicted Normalized by Body Mass Index
}

National Cancer Institute

\section{Source}

National Cancer Institute. AURC Infinity Predicted Normalized by Body Mass Index. NCI

Thesaurus. Code C92358.

The area under the urinary excretion rate curve (AURC) extrapolated to infinity, based on the last predicted excretion rate, divided by the body mass index. 